\title{
CoRoT sounds the stars: p-mode parameters of Sun-like oscillations on HD $49933^{\star}$
}

\author{
T. Appourchaux ${ }^{1}$, E. Michel ${ }^{2}$, M. Auvergne ${ }^{2}$, A. Baglin ${ }^{2}$, T. Toutain ${ }^{3}$, F. Baudin ${ }^{1}$, O. Benomar ${ }^{1}$, W. J. Chaplin ${ }^{3}$

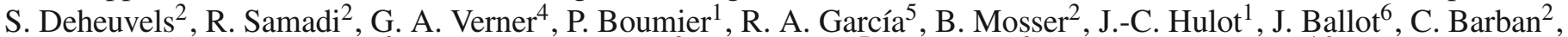 \\ Y. Elsworth ${ }^{3}$, S. J. Jiménez-Reyes ${ }^{8}$, H. Kjeldsen ${ }^{7}$, C. Régulo ${ }^{8}$, and I. W. Roxburgh ${ }^{4,2}$ \\ 1 Institut d'Astrophysique Spatiale, UMR8617, Université Paris XI, Bâtiment 121, 91405 Orsay Cedex, France \\ e-mail: Thierry.Appourchaux@ias.u-psud.fr \\ 2 LESIA, UMR8109, Université Pierre et Marie Curie, Université Denis Diderot, Observatoire de Paris, 92195 Meudon Cedex, \\ France \\ 3 School of Physics and Astronomy, University of Birmingham, Edgbaston, Birmingham B15 2TT, UK \\ 4 Astronomy Unit, Queen Mary, University of London Mile End Road, London E1 4NS, UK \\ ${ }_{6}^{5}$ Laboratoire AIM, CEA/DSM-CNRS - Univ. Paris 7 Diderot - IRFU/SAp, 91191 Gif-sur-Yvette Cedex, France \\ 6 Max Planck Institut für Astrophysik, Karl-Schwarzschild-Str. 1, Postfach 1317, 85741 Garching, Germany \\ 7 Danish AsteroSeismology Centre (DASC), Department of Physics and Astronomy, University of Aarhus, 8000 Aarhus C, Denmark \\ 8 Instituto de Astrofísica de Canarias, 38205, La Laguna, Tenerife, Spain
}

Received 30 May 2008 / Accepted 7 July 2008

\section{ABSTRACT}

Context. The first asteroseismology results from CoRoT are presented, on a star showing Sun-like oscillations. We have analyzed a 60 day lightcurve of high-quality photometric data collected by CoRoT on the F5 V star HD 49933. The data reveal a rich spectrum of overtones of low-degree $\mathrm{p}$ modes.

Aims. Our aim was to extract robust estimates of the key parameters of the p modes observed in the power spectrum of the lightcurve. Methods. Estimation of the mode parameters was performed using maximum likelihood estimation of the power spectrum. A global fitting strategy was adopted whereby 15 mode orders of the mode spectrum (45 modes) were fitted simultaneously.

Results. The parameter estimates that we list include mode frequencies, peak linewidths, mode amplitudes, and a mean rotational frequency splitting. We find that the average large frequency (overtone) spacing derived from the fitted mode frequencies is $85.9 \pm$ $0.15 \mu \mathrm{Hz}$. The frequency of maximum amplitude of the radial modes is at $1760 \mu \mathrm{Hz}$, where the observed rms mode amplitude is $3.75 \pm 0.23 \mathrm{ppm}$. The mean rotational splitting of the non-radial modes appears to be in the range $\approx 2.7 \mu \mathrm{Hz}$ to $\approx 3.4 \mu \mathrm{Hz}$. The angle of inclination offered by the star, as determined by fits to the amplitude ratios of the modes, appears to be in the range $\approx 50$ degrees to $\approx 62$ degrees.

Key words. methods: statistical - methods: observational - stars: oscillations

\section{Introduction}

CoRoT (COnvection, ROtation and planetary Transits) is a minisatellite $(<600 \mathrm{~kg})$ developed by the French space agency (Centre National d'Études Spatiales, CNES), with participation of the Science Program of ESA, Austria, Belgium, Brazil, Germany and Spain. Its heritage dates back to the proposal for a mission called EVRIS Etude de la Variabilité, de la Rotation et des Intérieurs Stellaires (Mangeney et al. 1981). The main scientific objectives of CoRoT are to use its high-performance photometry data to detect exoplanets, and to study the interiors of stars. A summary of the historical development of CoRoT may be found in Roxburgh (2006).

Exoplanets are detected by the small stellar dimming that results from the passage of a planet in front of its host star. Since the launch of CoRoT, in 2006 December, the satellite has already detected several exoplanets (Barge et al. 2008; Alonso et al. 2008; Bouchy et al. 2008). The internal structures and

* The CoRoT space mission, launched on 2006 December 27, was developed and is operated by the CNES, with participation of the Science Programs of ESA, ESA's RSSD, Austria, Belgium, Brazil, Germany and Spain. dynamics of stars are probed by asteroseismology (ChristensenDalsgaard 1984), whereby long timeseries are used to measure the photometric variations of stars resulting from standing waves in the stellar interiors. Stars like the Sun, which have sub-surface convection zones, display a rich spectrum of acoustic ( $p$-mode) oscillations. The fact that the numerous excited modes sample different interior volumes within the stars means that the internal structures can be probed and the stellar parameters constrained to levels of detail and precision that would not otherwise be possible. The Sun-like oscillations are stochastically excited and intrinsically damped by the convection. The photometric variations displayed by the low-degree (low- $l$ ), high-order overtones visible in main sequence stars have typical amplitudes of a few parts-per-million (ppm). These oscillations have periods of the order of minutes.

Photometric variations on a few-hundred stars will be monitored over the course of the CoRoT mission. Several of these target stars are expected to show Sun-like oscillations. In this paper, we report the first set of asteroseismology results obtained by CoRoT on a Sun-like oscillator, the F5 V main-sequence star HD 49933 previously observed by Mosser et al. (2005). The data come from the Initial Run of CoRoT. 
The layout of the rest of our paper is as follows. We begin in Sect. 2 with a brief recap of existing data on HD 49933, which come from non-asteroseismic observations. We then give details on the CoRoT data in Sect. 3, and include a summary of the lightcurve pipeline for the data. Section 4 describes in detail how we analyzed the lightcurve. We discuss important features of the power spectrum of the HD 49933 lightcurve in Sect. 4.1, and then spell out in Sect. 4.2 how we fitted p-mode peaks in the power spectrum to extract estimates of the mode parameters. Several important features of the p-mode spectrum of HD 49933 contrived to make this analysis very difficult. We present our original analysis strategy in Sect. 4.2.1, and then explain in Sect. 4.2.2 how (and why) this strategy was changed to circumvent the problems posed by the star's spectrum. The main results of the paper - our best-fitting estimates of the p-mode parameters - are presented in Sect. 5, and discussed in Sect. 6. We finish in Sect. 7 with a brief summary.

\section{Existing data on HD 49933}

\subsection{Temperature, luminosity and iron abundance}

HD 49933 is a main sequence F5 V star with an observed apparent visual magnitude $m_{\mathrm{V}}=5.77$. It has a measured iron abundance of -0.37 dex (Solano et al. 2005), making it metal poor compared to the Sun (and compared to another hot, Sunlike asteroseismic target, Procyon). The effective temperature is $T_{\text {eff }}=6780 \pm 130 \mathrm{~K}$, as given by Bruntt et al. (2008).

The absolute visual magnitude may be derived by making use of the revised Hipparcos parallax for the star, which is $33.7 \pm$ 0.4 mas (van Leeuwen 2007), giving $M_{\mathrm{V}}=3.408 \pm 0.026$. Use of an appropriate bolometric correction $(\mathrm{BC}=0.025 \pm 0.005$, from data in Bessell et al. 1998) gives an estimated luminosity of $\log L / L_{\odot}=0.53 \pm 0.01$.

\subsection{Mass, radius and $v \sin i$}

The radius of the star can then be deduced from $L$ and $T_{\text {eff }}$, i.e., $R / R_{\odot}=1.34 \pm 0.06$. This radius is quite close to the one inferred by Thévenin et al. (2006) ${ }^{1}$ using the Barnes-Evans surface brightness relation. A precise interferometric determination of the radius of HD 49933 is yet to be made.

An estimate of the mass of HD 49933 may be obtained by matching evolutionary tracks to the $L-T_{\text {eff }}$ error box in the colour-magnitude diagram. The resulting estimates give $M / M_{\odot} \sim 1.2$ (Mosser et al. 2005).

Another important parameter of interest to us in this paper is the measured $v \sin i$ of the star. Recent measurements of $v \sin i$ for HD 49933 range between $10.9 \mathrm{~km} \mathrm{~s}^{-1}$ as determined by Solano et al. (2005), and $9.5 \pm 0.3 \mathrm{~km} \mathrm{~s}^{-1}$ as determined by Mosser et al. (2005) (see also Gillon \& Magain 2006, for other estimates that lie between the values quoted here). The data for the Mosser et al. determination came from observations made by the HARPS spectrograph. The seismic run on HD 49933 showed important variations of the line bisector, which contributed significantly to the line broadening. While every effort was made to reduce the influence of these variations - stellar parameters were derived from spectra recorded during what seemed to be a stable phase of the star - the estimated $v \sin i$ may nevertheless be an overestimate of the true value.

\footnotetext{
${ }^{1} R / R_{\odot}=1.43 \pm 0.06$ without limb-darkening correction, note that there is a typographical error in the radius in that paper that has been corrected here.
}

\section{CoRoT observations}

CoRoT was launched on 2006 December 27 from the Baikonur cosmodrome on a Soyuz Fregat II-1b launcher. After an initial period of commissioning lasting just over one month CoRoT's observing sequence began, with a pointing direction close to the anticenter of the galaxy. This "Initial Run" lasted from 2007 February 6 to 2007 April 7, i.e., 60 days, with the principal target being HD 49933. The field selected for the Initial Run complies with the requirement for the Seismology Core Programme - that it should contain one main-sequence, Sun-like oscillator with apparent magnitude $m_{\mathrm{V}}<6-$ and also with the requirement for the Exoplanet Programme - that the density of stars should exceed 1300 stars per degree-squared (Michel et al. 2006).

A detailed description of how photometric data are extracted for the seismology field has already been presented in the CoRoT book (see Baglin 2006, and references therein). We give a brief summary of the main steps here.

The $27 \mathrm{~cm}$ diameter afocal telescope of CoRoT makes an image of the stellar field onto a four-frame transfer CCD, having $2048 \times 4096$ pixels. There are two CCDs dedicated to the seismology program. The images on these CCD are defocused over $\approx 400$ pixels in order to reduce the effect of spacecraft jitter on the photometry. For each run, five stellar windows (each occupying $50 \times 50$ pixels), five sky-reference windows, and two offset reference windows, are defined per CCD. Stellar fluxes are read every $1 \mathrm{~s}$, with a dead time of $0.206 \mathrm{sec}$ (implying a sampling cycle of 79.4\%). No flat field correction is performed, since pixel-to-pixel non-uniformities (PRNU) are less than $0.6 \%$. The pointing of the CoRoT platform is also very stable (measured performance jitter $<0.15$ arcsec rms). Photometric disturbance induced by the combined effect of the PRNU and pointing jitter amounts to less than 3 ppm rms.

Stellar fluxes are extracted on-board from each stellar window by performing aperture photometry, i.e., by summing all photoelectrons in a given window using an appropriate mask for the integration. A mean stellar flux of $7.6 \times 10^{6}$ electrons per integration was measured for HD 49933, against a mean level for the sky background of 21.5 counts. The total flux per $32 \mathrm{~s}$ sample is $\approx 244 \times 10^{6}$ electrons.

Once the aperture photometry data have been obtained several corrections are applied, the details of which may be found in Samadi et al. (2007a) and Baudin et al. (in preparation). Depointing problems can occur during periods when there are sunlight eclipses, resulting in the target star drifting either toward the edge of the aperture photometry mask or in a few cases even beyond the edge of the mask. These effects are corrected with a model, devised by De Oliveira Fialho \& Auvergne (2006) (see also De Oliveira Fialho et al. 2007; Drummond et al. 2006), which takes full account of the point spread function, of the masks, and of the spacecraft pointing.

After these corrections have been made, the resulting lightcurves are resampled onto a regular cadence in the heliocentric frame, with one measurement every 32 s. These "Level 2" lightcurves show near-continuous coverage over the 60 days of the Initial Run, with only a small number of gaps that result mainly from the passage of CoRoT across the South Atlantic Anomaly. These short gaps were filled by suitable interpolation. The duty cycle for HD 49933 before interpolation was $90 \%$. 


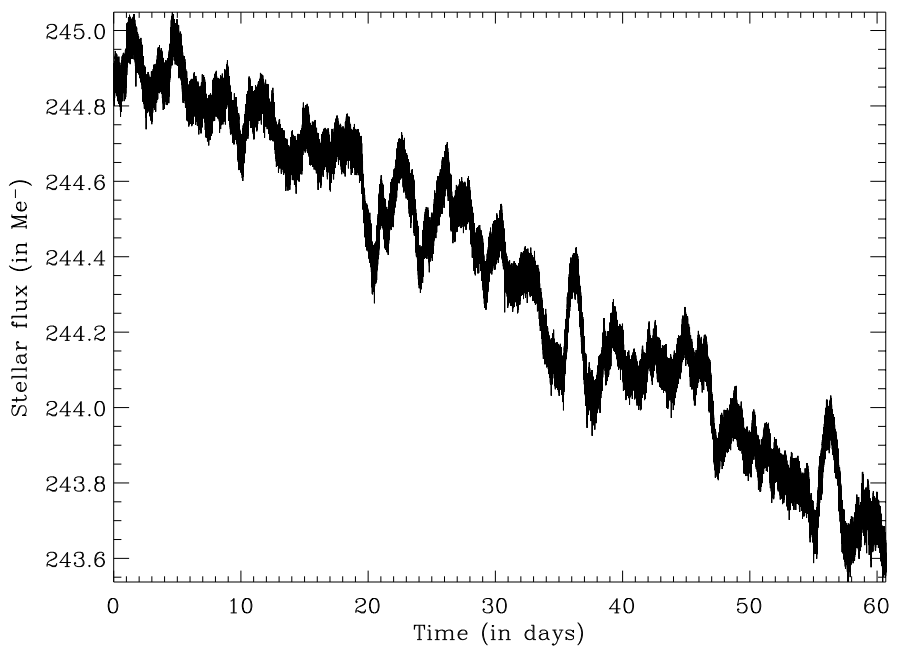

Fig. 1. Level-2 lightcurve of HD 49933. The variations are typically 2000 ppm peak-to-peak.

\section{Data analysis}

\subsection{Time series and power spectrum}

Figure 1 shows gap-filled Level-2 lightcurve. The quasi-periodic variations visible in the lightcurve are due to rotational modulation of active-region features (see below). The Level-2 lightcurve was high-pass filtered for subsequent analysis by subtraction of a best-fitting second-order polynomial in time that removed the persistent downward trend seen over the 60 days of observation. This yielded a residual lightcurve. The top panel of Fig. 2 shows the power spectrum of the residual lightcurve, computed using the Fast Fourier Transform. The power spectrum was calibrated such that the total power integrated from zero to twice the Nyquist frequency was equal to the variance of the residual lightcurve. The impact of the gap interpolation on the power spectrum will depend upon frequency. The typical length of the gaps is about $9 \mathrm{~min}$, thereby producing a a negligible change in the power spectrum for periods longer than $18 \mathrm{~min}$ $(v<925 \mu \mathrm{Hz})$, and a reduced power by $10 \%$ for periods shorter than $9 \min (v>1850 \mu \mathrm{Hz})$.

Prominent features due to both stellar and orbital signatures are present in the power spectrum shown in the top panel of Fig. 2. There are peaks at very low frequencies due to stellar activity, which we come back to below. The background rises in the lower-frequency part of the spectrum, with contributions from granulation and active-region noise. A large peak is present at $161.7 \mu \mathrm{Hz}$, which lies at the orbital period of the satellite. And there is a broader cluster of power due to the p modes, which is centered on $1800 \mu \mathrm{Hz}$. The bottom panel of Fig. 2 shows the range in frequency occupied by the $\mathrm{p}$ modes. Here, the spectrum has been smoothed with a boxcar of width $20 \mu \mathrm{Hz}$. Over 20 orders (overtones) of the low- $l$ modes can be clearly seen.

The high-frequency noise power spectral density - which gives a measure of the photon shot noise - is about $0.15 \mathrm{ppm}^{2} / \mu \mathrm{Hz}$, or about $15 \%$ higher than expectations based on the apparent brightness of the target and the predicted performance of the instrument. The main source of this extra noise is due to the effects of spacecraft jitter on the aperture photometry.

The low-frequency part of the spectrum contains a prominent, narrow peak at $3.4 \mu \mathrm{Hz}$, as shown in Fig. 3. The second and fourth harmonics of this peak are also very apparent in the power spectrum. The third harmonic is not observed. We suggest that the strong, $3.4 \mu \mathrm{Hz}$ peak (corresponding to a period of 3.4 days)
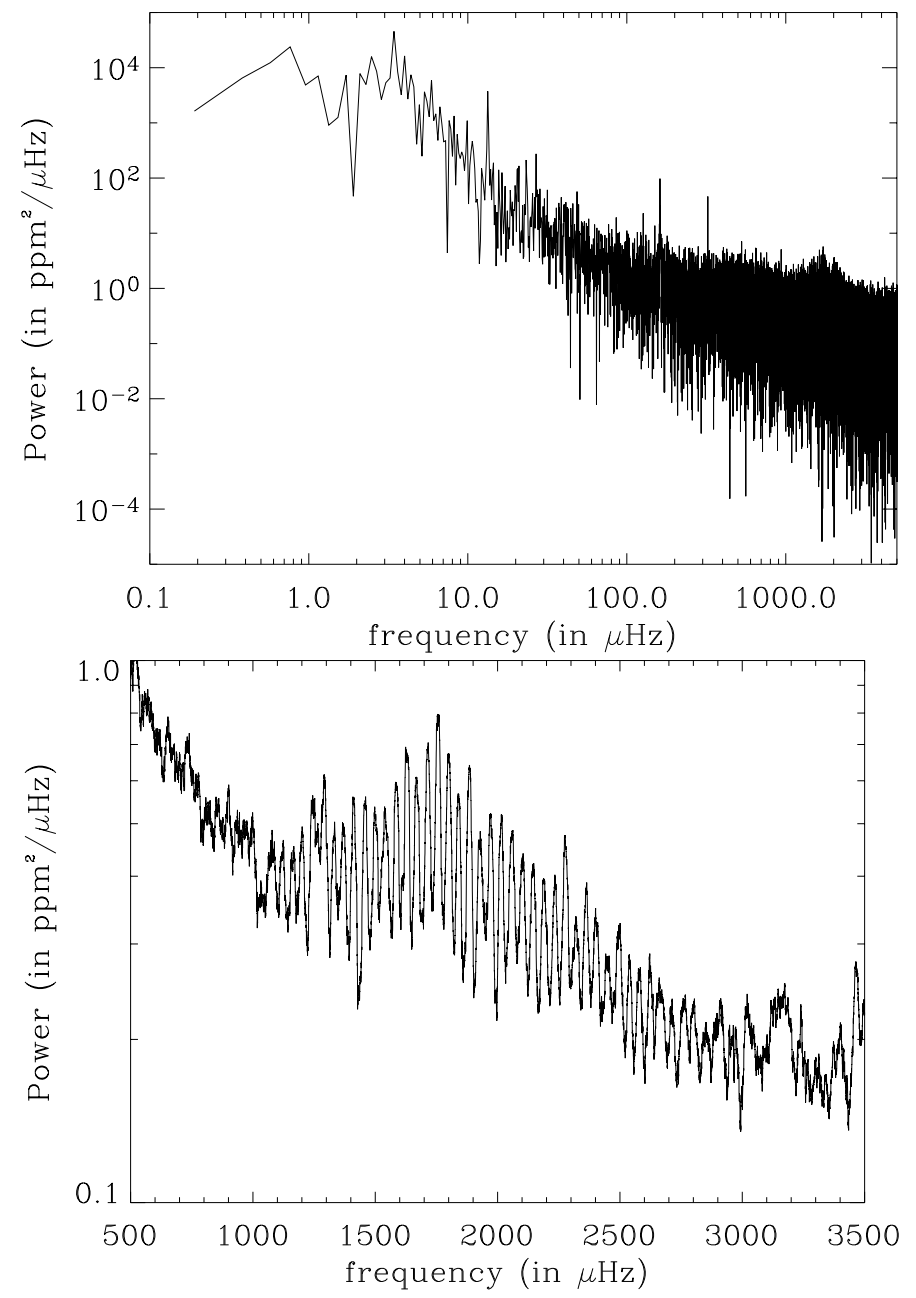

Fig. 2. Top panel: Raw power spectrum as a function of frequency, showing peaks due to stellar activity (see also Fig. 3), a strong peak due to the orbital period of the satellite (at $161.7 \mu \mathrm{Hz}$ ), and peaks due to p modes (centered on $1800 \mu \mathrm{Hz}$ ). Bottom panel: Power spectrum, after smoothing with a $20 \mu \mathrm{Hz}$ boxcar, showing the range in frequency occupied by the p modes. Modes are clearly visible up to $2800 \mu \mathrm{Hz}$. The spectral resolution is about $0.19 \mu \mathrm{Hz}$.

is the first harmonic of the surface rotation period (more specifically, some suitably weighted mean rotation period of those latitude bands that are occupied by the active-region signal). The presence of the first, second and fourth harmonics is consistent with the findings of Clarke (2003), who examined analytically the simple case of the rotational modulation of a lightcurve due to a single equatorial co-rotating spot on a star inclined at 90 degrees. We carried out additional simulations which showed that the conclusions are not modified significantly when the inclination differs from 90 degrees, or when there are several spots (we note that in some cases the third harmonic was observed, though at much weaker levels than the other harmonics). Clarke (2003) demonstrated that all the harmonics of the rotational period are present in observations made in Doppler velocity, while some harmonics are suppressed in intensity. Interpretation of our low-frequency spectrum therefore suggests a surface rotation period of 3.4 days.

Given that we already have estimates of $R$ (Thévenin et al. 2006) and $v \sin i$ (see Sect. 2 above), we can in principle then obtain an estimate of the angle of inclination, $i$, offered by the 


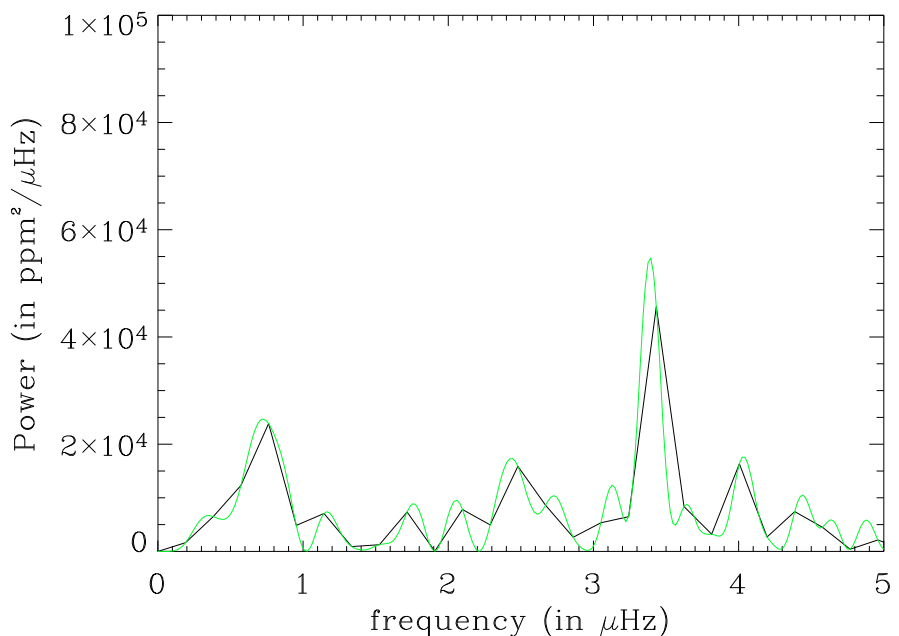

Fig. 3. Raw power spectrum (black), oversampled power spectrum by a factor 10 (green) both at very low frequencies, and both showing a strong peak at $3.4 \mu \mathrm{Hz}$, arising from rotational modulation of active-region features on the surface of HD 49933 (see text).

star. The measured stellar rotation period, quoted above, leads to an estimated inclination between 22 and 30 degrees.

The inclination is an important parameter for analysis of $p$ modes in the power spectrum. This is because the inclination affects the relative amplitudes of rotationally split components in non-radial modes. Use of poor estimates of the inclination when modeling the modes can result in significant biases in some of the mode parameters, in particular in the frequency splittings (e.g., see Ballot et al. 2006, 2008). However, the inclination can in principle be constrained by asteroseismology, provided the mode amplitude ratios can be measured reliably (Gizon \& Solanki 2003).

\subsection{Extraction of mode parameters by Maximum Likelihood Estimation}

The mode parameters were extracted with analysis techniques that have been applied extensively since the late 1980s to data on the stochastically excited, damped p modes of the Sun (Duvall $\&$ Harvey 1986). The method consists in fitting the power spectrum using maximum likelihood estimation (MLE) (Anderson et al. 1990). MLE has been applied widely in the helioseismic community, both for analyzing low- $l$ modes, and medium and higher-l modes (e.g., see Schou 1992; Appourchaux et al. 1998; Chaplin et al. 2006; and references therein). The formal uncertainties associated with MLE are very well understood, as explained by Libbrecht (1992), Toutain \& Appourchaux (1994) and Appourchaux et al. (1998).

The best-fitting mode parameter estimates given by MLE are robust, in that those estimates are asymptotically unbiased (in the statistical sense) and of minimum variance (lower bounds given by the Cramer-Rao criterion; see Toutain \& Appourchaux 1994). That said, it is important to recognize that while statistical biases may be absent, other biases may not, e.g., those arising from inappropriate modelization of the shapes of the mode peaks, or of the background noise.

Before we fitted the power spectrum, we first checked that use of the interpolated points in the lightcurve gave negligible correlations between frequency bins in the power spectrum, which meant we could treat the bins as being statistically

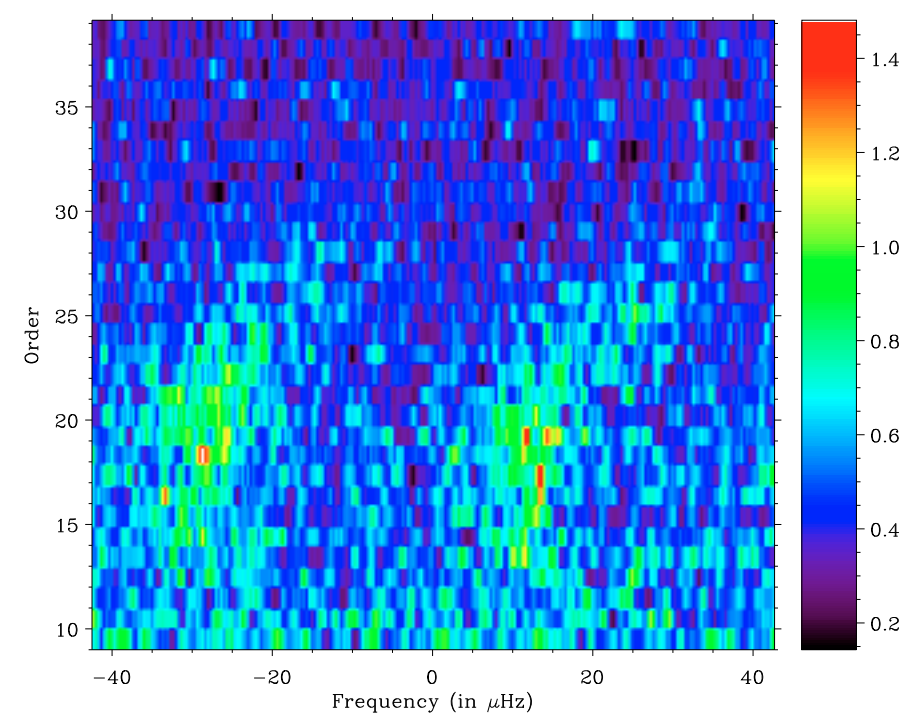

Fig. 4. Echelle diagram computed for a large separation of $85 \mu \mathrm{Hz}$. The diagram is smoothed over $0.8 \mu \mathrm{Hz}$ (4 bins). The order numbering was chosen to match numbering given later in the mode frequency table (Table 1; see also Sect. 5). Vertically aligned ridges of power, due to the $l=0$ and $l=2$ modes, and the $l=1$ modes, are clearly visible. However, the identification of the ridges is far from evident.

independent. This is an important criterion for application of the MLE.

\subsubsection{The CoRoT recipe}

MLE analysis methodologies for the CoRoT asteroseismology data were tested in a series of hare-and-hounds exercises conducted by the Data Analysis Team (DAT) of the Seismology Working Group (Appourchaux et al. 2006a). One of the main objectives of these exercises was to develop a formal CoRoT analysis recipe for extracting estimates of $\mathrm{p}$-mode parameters from power spectra of Sun-like stars observed by CoRoT. Such a recipe would allow "CoRoT reference" mode-parameter sets to be released on stars; and the recipe would be developed, and fully documented, in such a way as to be reproducible by other scientists.

Soon after the Initial Run CoRoT data on HD 49933 were available, it became clear that important characteristics of the mode spectrum presented what was almost a worse-case scenario for the analysis. This forced us to revisit the CoRoT recipe, and to move towards a more sophisticated analysis of the power spectrum to resolve some of the problems posed by the data. The revised analysis strategy is outlined in Sect. 4.2.2 below. To set that strategy in context, it behoves us to summarize first the key elements of the original CoRoT recipe (see also Appourchaux et al. 2006a).

The large separation $\Delta v$ is estimated from an echelle diagram of the power spectrum in which ridges are tagged as being due to the $l=0$ and $l=2$ modes or the $l=1$ modes. Then, MLE is applied to the power spectrum to extract estimates of the mode parameters. Modes are fitted as Lorentzian functions in a window $\Delta v / 3$ wide. Non-radial modes are fitted with a common linewidth and symmetrical splitting in frequency. The relative amplitudes of peaks in non-radial mode multiplets are described by the intensity visibilities in Gizon \& Solanki (2003). The stellar angle of inclination is derived from the ratios of the bestfitting amplitudes. Finally, the background is considered to be flat in the fitting window. 

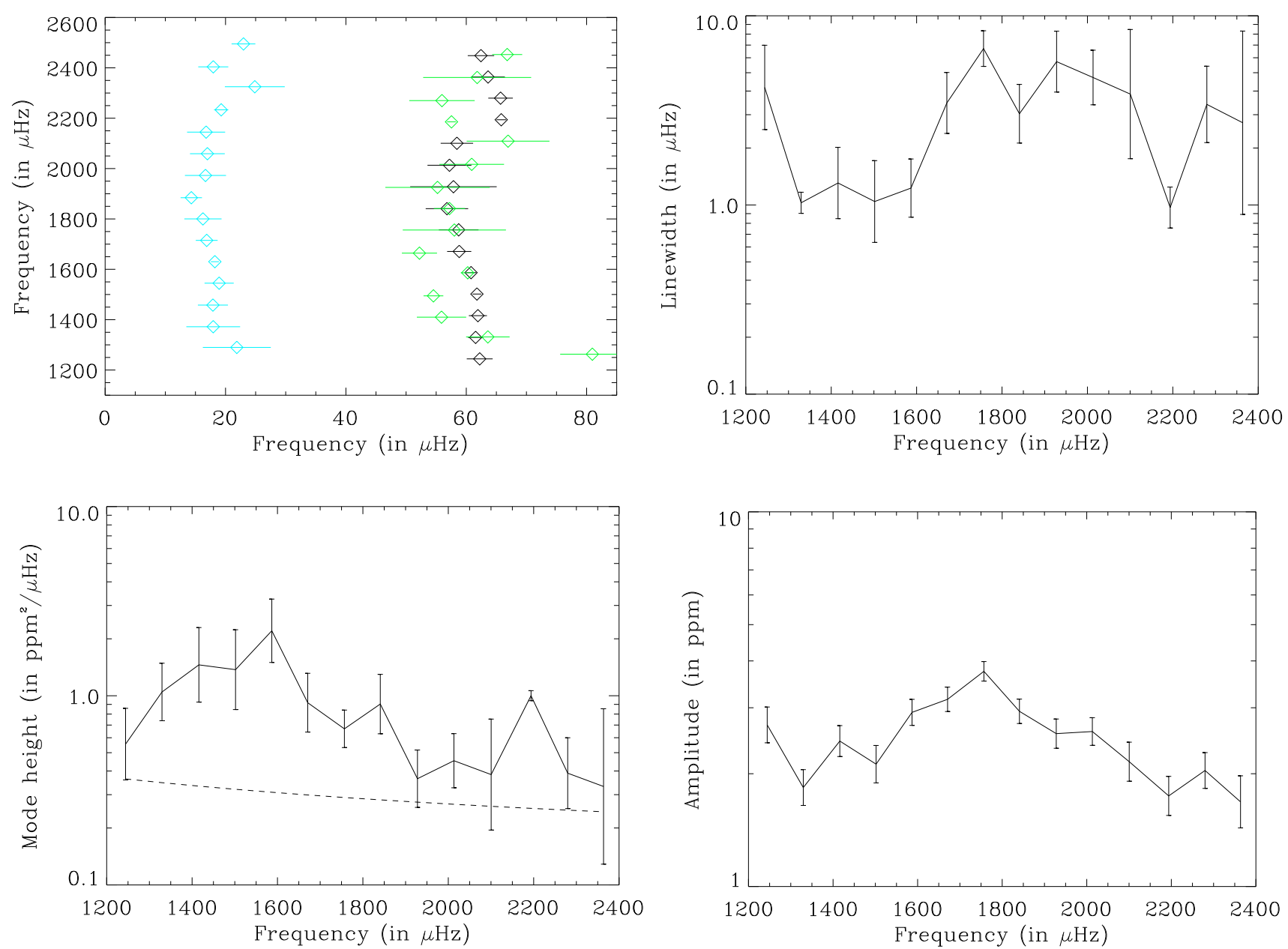

Fig. 5. Top left-hand panel: echelle diagram made using the best-fitting mode frequencies from Table $1(l=0$ in black, $l=1$ in cyan, $l=2$ in green); the error bars are the $3 \sigma$ values. Top right-hand panel: $l=0$ mode linewidths from Table 2 (error bars are $1 \sigma$ values). Bottom left-hand panel: $l=0$ mode heights from Table 2 (error bars are $1 \sigma$ values). The dashed line shows the best-fitting background from the Reference fit. Bottom right-hand panel: $l=0$ mode amplitudes from Table 3 (error bars are $1 \sigma$ values).

The recipe, as described above, was applied to the power spectrum of HD49933. We now go on to discuss the problems that the power spectrum presented for this analysis.

\subsubsection{Extraction of mode parameters for HD 49933}

Figure 4 is the echelle diagram of the power spectrum, which shows the expected, near-vertically aligned ridges of power. The recipe gives a robust, and well-constrained estimate of the mean large frequency spacing of HD 49933, of $\Delta v \sim 85 \mu \mathrm{Hz}$. It is when we move to the degree identification that we run into immediate problems. As should be evident from a simple visual inspection of Fig. 4 it is next to impossible to tell one ridge apart from the other. If we do not know which ridge is due to the $l=0$ and $l=2$ modes, and which ridge is due to the $l=1$ modes, there will be obvious problems for the MLE fitting since we need a priori knowledge of the ridge identification to allow us to set up the mode-fitting models correctly.

One potential way out of the problem is to make a comparative test of the two possible ridge identifications. MLE fits are made for the two scenarios. The likelihood ratio test is then applied to the best-fitting MLE data to find which best-fitting model is the more likely of the two. The likelihood ratio test requires that we compute the ratio of the maximized likelihoods of each model, which come from the MLE fits (i.e. from the models that maximize the likelihood of the data).

Let us tag the two possible ridge identification scenarios by the letters $\mathrm{A}$ and $\mathrm{B}$. In scenario $\mathrm{A}$, the peak at frequency $\sim 1501 \mu \mathrm{Hz}$ is identified as an $l=0$ mode. In scenario $\mathrm{B}$, the same peak is assumed to be an $l=1$ mode. We must then test the two models of the spectrum, $M_{\mathrm{A}}$ and $M_{\mathrm{B}}$. The likelihood ratio is given by $\mathcal{L}_{\mathrm{r}}=p\left(\right.$ data $\left.\mid M_{\mathrm{A}}\right) / p\left(\right.$ data $\left.\mid M_{\mathrm{B}}\right)$. The posterior probability of model $\mathrm{A}$ is related directly to the likelihood ratio by $p\left(\mathrm{M}_{\mathrm{A}} \mid\right.$ data $)=\left(1+\mathcal{L}_{\mathrm{r}}^{-1}\right)^{-1}$. So, a very high likelihood ratio will imply a high posterior probability for model $M_{\mathrm{A}}$ versus model $M_{\mathrm{B}}$.

This is the only way we have to make an objective judgment regarding the relative merits of the two possible ridge identifications that is not based in any way on our own prejudices. However, it is important to remember that a higher likelihood does not mean that a given model is physically more meaningful, rather, it means the model is statistically more likely. Application of prejudice and a priori knowledge invokes a Bayesian approach to the problem. It was decided that such an approach could be used, but only after the more classical approach outlined above had been applied first (see Appourchaux 2008 for possible Bayesian approaches). 
In order to obtain the maximized likelihoods needed for the likelihood-ratio test, we first had to perform MLE fitting of the power spectrum. This turned out to be very difficult for several reasons. First, the height-to-background ratio is rather low $(<10)$. Secondly, the mode peaks have large widths in the power spectrum (typically several micro-Hertz), which presents problems due to severe blending of nearby mode components. Thirdly, the rotational frequency splittings, as deduced from Fig 3, are likely to be close to the surface rotation of $3.4 \mu \mathrm{Hz}$. This value is comparable to the typical widths shown by the peaks, and also the small frequency spacing between the $l=0$ and $l=2$ modes (See also Appourchaux et al. 2006b). Both coincidences further compound the problems caused by mode blending. Finally, modes show significant power over a wide range in frequency because of the large widths (and splittings). Quantitative checks imply that the recipe fitting window, which has width $\Delta v / 3$, is not wide enough to get robust estimates of the power falling in the wings of the mode peaks. We very quickly realized that the basic CoRoT recipe as listed above was not properly adapted to the case of HD 49933.

In an attempt to alleviate the problems, we began by widening the basic fitting-window width from $\Delta v / 3$ to $\Delta v$. This meant that each fitting window would now include three visible modes: an $l=0$, an $l=1$, and an $l=2$ mode. However, when we then applied the likelihood ratio test, we found that the test privileged either scenario A or scenario B dependent upon the location of the $l=1$ mode in the fitting window. This unsatisfactory situation with regard to the likelihood-ratio test resulted from the background noise determination interfering with the mode linewidth determination.

We eventually found that by increasing the fitting window to a width of $15 \Delta v$ - meaning that 15 orders are fitted simultaneously (a so-called "global fitting") - we could obtain consistent results from the likelihood-ratio test, which were obviously insensitive to the location of the modes in the window. Once this general fitting strategy had been chosen, it was implemented in slightly different ways (with differences in the background modeling, inclination angle determination, and linewidth assignment; and in the numbers of parameters freely fitted or kept fixed). All fitting variants gave consistent results for the likelihood ratio test.

We settled on the following detailed strategy, which we call the global Reference fit $^{2}$ :

- All free parameters of the global fitting model were varied, and optimized, simultaneously;

- The fitting was performed over 15 orders, with three frequencies per order, i.e., $l=0,1$ and 2;

- Mode peaks were described by Lorentzian functions;

- A single height parameter was fitted to modes in each order. The ratio of the mode heights was fixed at 1.5 for $l=1$ over $l=0$, and 0.5 for $l=2$ over $l=0$. These values include a correction for limb-darkening, performed in the manner described by Chaplin et al. (2008);

- Relative amplitudes of peaks in the non-radial mode multiplets were described by the intensity visibilities calculated for adiabatic fluctuations (Gizon \& Solanki 2003); the angle of inclination fixes these ratios, and the angle was estimated by fitting simultaneously the amplitude ratios of all non-radial modes in the 15 target orders;

- A single linewidth parameter was fitted to modes in each order;

${ }^{2}$ Roca Cortés et al. (1999) performed the first global fit on a solar power spectrum.
- Non-radial modes were assumed to show symmetric splitting in frequency, and a single rotational splitting parameter was fitted to all non-radial modes in the 15 target orders; and

- The background model used a power law to model the granulation component Harvey (1985). The exponent in the power law was allowed to vary, and optimized during fitting. There was also a second, flat component to model the photon shot noise. All free parameters associated with the background were fitted simultaneously along with the mode parameters.

Guess parameters for the $l=0$ and $l=1$ frequencies were set to the frequencies given by fitting individual windows of width $\Delta v$, while the $l=2$ frequencies were set $5 \mu \mathrm{Hz}$ below the frequencies of the $l=0$ modes. Guess parameters for the mode heights were all set to the same value, this being one-quarter of the maximum power spectral density in the entire global fitting range. Guess parameters for the noise background were estimated from the fits performed on individual windows of width $\Delta v$. The firstguess splitting was set to $3.4 \mu \mathrm{Hz}$ (Fig. 3), and the first-guess angle of inclination was set to 45 degrees. In total, 80 parameters were varied and optimized by the Reference fit. After convergence, the full Hessian matrix was computed to return parameter uncertainties and parameter covariances. The covariances are important for estimation of uncertainties on the estimated mode amplitudes, which are proportional to the square root of the product of the height and linewidth parameters. The heights and linewidths are strongly correlated. The mode parameter estimates and uncertainties that we give later, in Tables 1, 2, and 3, come from the Reference fit.

Results obtained from the Reference fit were cross-checked by six other fitters, who implemented their own global fitting codes. Differences between codes were present in:

- The number of linewidth parameters fitted per order (e.g., linewidth could be different for $l=0, l=1$ and $l=2$ modes);

- The practical details of the way in which the parameters were optimized, e.g., fit all parameters simultaneously; use an iterative approach (as in Gelly et al. 2002), where parameters are optimized in batches; or fix some parameters at their input values;

- The background noise model (e.g. polynomial functions, or the Harvey model Harvey 1985); and

- The global fitting range in frequency.

The results from the global fits are presented hereafter.

\section{Results from global fits}

Results from the global fits showed that ridge identification scenario A was clearly privileged by the likelihood ratio test. For example, the Reference fit gave a likelihood ratio of 1000 for identification A against identification B. Given the assumption we made about the models, the posterior probability associated with this likelihood ratio is $p\left(M_{\mathrm{A}} \mid\right.$ data $)=99.9 \%$.

Best-fitting mode parameters returned by the Reference fit are listed in Table 1 (frequencies), Table 2 (heights and linewidths), and Table 3 (amplitudes). Fits are for the preferred ridge identification scenario $\mathrm{A}$. The heights, linewidths and amplitudes may be regarded as being those of the $l=0$ modes.

We make a few important remarks regarding the data in the tables. First, the orders of the modes were chosen so as to give the closest match to the frequencies of a full evolutionary model of HD 49933 made by one of us (IWR). Secondly, we have marked with filled circles those mode parameters for which at least six of the seven fitters agreed on the value of the parameter 
Table 1. Mode frequencies of HD 49933, returned by the Reference fit. Data marked with filled circles indicate where at least six of the seven fitters agreed on the value of the parameter to within $1 \sigma$. Details are given in those cases where disagreement was noted at $>1 \sigma$.

\begin{tabular}{|c|c|c|c|c|c|c|}
\hline 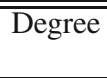 & Order & "Frequency (in $\mu \mathrm{Hz}$ ) & "Uncertainty (in $\mu \mathrm{Hz}$ ) & "Height-to-background ratio & $\begin{array}{l}\text { Agreement } \\
\quad<1 \sigma\end{array}$ & $\begin{array}{c}\text { Disagreement } \\
\text { value }\end{array}$ \\
\hline 0 & 14 & 1244.23 & 0.72 & 1.5 & $\bullet$ & \\
\hline 0 & 15 & 1329.55 & 0.42 & 3.0 & & $>11 \sigma$ \\
\hline 0 & 16 & 1415.93 & 0.50 & 4.4 & - & \\
\hline 0 & 17 & 1501.77 & 0.25 & 4.3 & $\bullet$ & \\
\hline 0 & 18 & 1586.79 & 0.28 & 7.1 & - & \\
\hline 0 & 19 & 1670.83 & 0.67 & 3.1 & - & \\
\hline 0 & 20 & 1756.74 & 1.10 & 2.3 & - & \\
\hline 0 & 21 & 1840.79 & 1.18 & 3.2 & - & \\
\hline 0 & 22 & 1927.87 & 2.40 & 1.3 & - & \\
\hline 0 & 23 & 2013.21 & 1.22 & 1.7 & - & \\
\hline 0 & 24 & 2100.45 & 0.89 & 1.5 & & $>3.3 \sigma$ \\
\hline 0 & 25 & 2193.45 & 0.27 & 3.9 & & $>4.3 \sigma$ \\
\hline 0 & 26 & 2279.70 & 0.68 & 1.6 & - & \\
\hline 0 & 27 & 2363.62 & 0.93 & 1.4 & & $>7.2 \sigma$ \\
\hline 1 & 14 & 1289.87 & 1.88 & 2.3 & $\bullet$ & \\
\hline 1 & 15 & 1372.96 & 1.49 & 4.5 & & $>2.0 \sigma$ \\
\hline 1 & 16 & 1457.91 & 0.83 & 6.6 & - & \\
\hline 1 & 17 & 1544.91 & 0.83 & 6.4 & - & \\
\hline 1 & 18 & 1630.23 & 0.22 & 10.7 & - & \\
\hline 1 & 19 & 1714.87 & 0.61 & 4.6 & - & \\
\hline 1 & 20 & 1800.25 & 1.03 & 3.5 & $\bullet$ & \\
\hline 1 & 21 & 1884.31 & 0.59 & 4.8 & $\bullet$ & \\
\hline 1 & 22 & 1972.67 & 1.14 & 2.0 & - & \\
\hline 1 & 23 & 2058.99 & 0.96 & 2.5 & - & \\
\hline 1 & 24 & 2144.77 & 1.05 & 2.2 & - & \\
\hline 1 & 25 & 2233.28 & 0.39 & 5.9 & - & \\
\hline 1 & 26 & 2324.87 & 1.66 & 2.4 & & $>2.4 \sigma$ \\
\hline 1 & 27 & 2403.96 & 0.83 & 2.0 & - & \\
\hline 2 & 13 & 1262.95 & 1.78 & 0.8 & $\bullet$ & \\
\hline 2 & 14 & 1331.59 & 1.20 & 1.5 & & $>2.4 \sigma$ \\
\hline 2 & 15 & 1409.89 & 1.37 & 2.2 & & $>2.0 \sigma$ \\
\hline 2 & 16 & 1494.54 & 0.55 & 2.1 & & $>3.0 \sigma$ \\
\hline 2 & 17 & 1586.24 & 0.38 & 3.6 & & $>4.1 \sigma$ \\
\hline 2 & 18 & 1664.22 & 0.97 & 1.5 & & $>2.7 \sigma$ \\
\hline 2 & 19 & 1756.02 & 2.86 & 1.1 & - & \\
\hline 2 & 20 & 1841.21 & 0.86 & 1.6 & $\bullet$ & \\
\hline 2 & 21 & 1925.22 & 2.89 & 0.7 & $\bullet$ & \\
\hline 2 & 22 & 2016.91 & 1.79 & 0.8 & $\bullet$ & \\
\hline 2 & 23 & 2108.94 & 2.29 & 0.7 & & $>2.4 \sigma$ \\
\hline 2 & 24 & 2185.54 & 0.25 & 2.0 & & $>5.2 \sigma$ \\
\hline 2 & 25 & 2269.96 & 1.81 & 0.8 & & $>3.0 \sigma$ \\
\hline 2 & 26 & 2361.81 & 2.99 & 0.7 & & $>1.4 \sigma$ \\
\hline
\end{tabular}

Table 2. Mode height and linewidth parameters for HD 49933, given by the Reference fits. Values are relevant to $l=0$. Data marked with filled circles indicate where at least six of the seven fitters agreed on the value of the parameter to within $1 \sigma$. Details are given in those cases where disagreement was noted at $>1 \sigma$. The mode heights are typically underestimated by about $5 \%$ due to the interpolation of the gaps.

\begin{tabular}{|c|c|c|c|c|c|c|c|}
\hline Degree & Order & $\begin{array}{l}\text { Mode height } H \\
\left(\text { in } \mathrm{ppm}^{2} / \mu \mathrm{Hz} \text { ) }\right.\end{array}$ & $\begin{array}{c}\text { Uncertainty } \\
\left(\text { in } \mathrm{ppm}^{2} / \mu \mathrm{Hz} \text { ) }\right.\end{array}$ & $\begin{array}{l}\text { Linewidth } \Gamma \\
\text { (in } \mu \mathrm{Hz} \text { ) }\end{array}$ & $\begin{array}{l}\text { Uncertainty } \\
\text { (in } \mu \mathrm{Hz} \text { ) }\end{array}$ & $\begin{array}{l}\text { Agreement } \\
\quad<1 \sigma\end{array}$ & $\begin{array}{l}\text { Disagreement } \\
\text { value }\end{array}$ \\
\hline 0 & 14 & 0.55 & $+0.30 /-0.20$ & 4.18 & $+2.80 /-1.68$ & $\bullet$ & \\
\hline 0 & 15 & 1.05 & $+0.44 /-0.31$ & 1.03 & $+0.14 /-0.12$ & & $>2.5 \sigma$ on $\log \Gamma, H$ \\
\hline 0 & 16 & 1.46 & $+0.84 /-0.53$ & 1.31 & $+0.71 /-0.46$ & - & \\
\hline 0 & 17 & 1.37 & $+0.85 /-0.53$ & 1.04 & $+0.67 /-0.41$ & & $>2.5 \sigma$ on $\log \Gamma, H$ \\
\hline 0 & 18 & 2.20 & $+1.04 /-0.71$ & 1.23 & $+0.52 /-0.37$ & $\bullet$ & \\
\hline 0 & 19 & 0.92 & $+0.39 /-0.28$ & 3.46 & $+1.55 /-1.07$ & • & \\
\hline 0 & 20 & 0.67 & $+0.17 /-0.14$ & 6.71 & $+1.62 /-1.31$ & - & \\
\hline 0 & 21 & 0.90 & $+0.39 /-0.27$ & 3.03 & $+1.30 /-0.91$ & - & \\
\hline 0 & 22 & 0.36 & $+0.15 /-0.11$ & 5.72 & $+2.56 /-1.77$ & • & \\
\hline 0 & 23 & 0.45 & $+0.18 /-0.13$ & 4.71 & $+1.86 /-1.33$ & $\bullet$ & \\
\hline 0 & 24 & 0.38 & $+0.37 /-0.19$ & 3.85 & $+4.61 /-2.10$ & • & \\
\hline 0 & 25 & 1.00 & $+0.06 /-0.06$ & 0.97 & $+0.27 /-1.26$ & & $>7.0 \sigma$ on $\log \Gamma, H$ \\
\hline 0 & 26 & 0.39 & $+0.21 /-0.14$ & 3.40 & $+2.01 /-1.26$ & - & \\
\hline 0 & 27 & 0.33 & $+0.52 /-0.20$ & 2.72 & $+5.58 /-1.83$ & - & \\
\hline
\end{tabular}


Table 3. rms mode amplitude parameters for HD 49933, given by the product $\pi H \Gamma$, with the height and linewidth parameters coming from the Reference fits. Values are relevant to $l=0$. Data marked with filled circles indicate where at least six of the seven fitters agreed on the value of the parameter to within $1 \sigma$. (See Table 2 for details on those cases where disagreement in the height and linewidth parameters was noted at $>1 \sigma$.) The rms mode amplitudes are typically underestimated by about $2.5 \%$ due to the interpolation of the gaps.

\begin{tabular}{cccc}
\hline \hline Order & $\begin{array}{c}\text { rms mode amplitude } \\
\sqrt{\pi H \Gamma}(\text { in ppm) }\end{array}$ & $\begin{array}{c}\text { Uncertainty } \\
\text { (in ppm) }\end{array}$ & Agreement \\
\hline 14 & 2.70 & 0.30 & $\bullet$ \\
15 & 1.84 & 0.20 & \\
16 & 2.44 & 0.23 & $\bullet$ \\
17 & 2.12 & 0.24 & \\
18 & 2.92 & 0.23 & $\bullet$ \\
19 & 3.16 & 0.24 & $\bullet$ \\
20 & 3.75 & 0.23 & $\bullet$ \\
21 & 2.94 & 0.23 & $\bullet$ \\
22 & 2.56 & 0.22 & $\bullet$ \\
23 & 2.59 & 0.23 & $\bullet$ \\
24 & 2.15 & 0.26 & $\bullet$ \\
25 & 1.75 & 0.21 & \\
26 & 2.04 & 0.22 & $\bullet$ \\
27 & 1.68 & 0.27 & $\bullet$ \\
\hline
\end{tabular}

to within $1 \sigma$ (significances judged by the formal fitting uncertainties). Most of the listed mode parameters fall into this category (though the more widespread disagreement in the $l=2$ frequencies is to be noted). In most cases all fitters agreed to within $1 \sigma$. We give details on the level of disagreement for those parameters where this was not the case. Those who intend to make use of our mode parameters should bear the listed discrepancies in mind, and we advise use only of those parameters validated by the $1 \sigma$ threshold. Thirdly, even though 15 orders were fitted, robust parameter estimates were returned on only 14 orders. Fourthly, a note on the mode amplitudes. The amplitudes in Table 3 were estimated from the products $\sqrt{\pi H \Gamma}$, and estimation of the uncertainties took into account the strong anti-correlation between the height and linewidth parameters.

We found that the global fits tended to converge on one of two different groups of rotational splitting values dependent on the chosen model: one grouping at about $2.7 \mu \mathrm{Hz}$, and another grouping at about $3.4 \mu \mathrm{Hz}$. Similarly, the different fitting codes could lock onto either one of the two solutions if we altered the background fitting model. Further investigation revealed that the likelihood for the splitting parameter did not have a single (optimal) maximum, but several maxima. The complicated nature of the likelihood was caused by a combination of several effects: the large linewidths, the similarity of the splittings to those linewidths and to the small frequency spacings between $l=0$ and $l=2$, and the low height-to-background ratio of the mode peaks.

The best-fitting splitting given by the Reference fit was $2.66 \pm 0.02 \mu \mathrm{Hz}$, while the best-fitting inclination angle was $55.7 \pm 3.8$ degrees. This inclination angle is not in agreement with the inclination angle derived in Sect. 4.1 from the $v \sin i$ data.

The best-fitting mode parameters of the $l=0$ and $l=$ 1 modes have clearly been estimated in a robust manner ( $1 \sigma$ agreement for 24 modes out of 28 ), whereas the agreement for the $l=2$ modes is not so strong. The uncertainties given for these parameters are slightly larger than the lower-bound expectations given by Libbrecht (1992) and by
Toutain \& Appourchaux (1994); but this is to be expected given the low height-to-background ratios and the large linewidths of the mode peaks.

\section{Discussion}

The top left-hand panel of Fig. 5 is an echelle diagram made from the best-fitting frequencies presented in Table 1 . The plot shows the difficulty of obtaining a proper identification of the $l=$ 0 and $l=2$ modes under circumstances (e.g., see Appourchaux et al. 2006b) where the linewidths are large, and comparable in magnitude to the mode frequency splittings. Indeed, the fitted $l=2$ modes do not always lie on the low-frequency side of the $l=0$ modes. In this paper, we deliberately chose not to rely on information from stellar evolution theory in rejecting or assessing the results of our fits. Some a priori constraints could in principle be applied to, for example, regularize the fits to force the small frequency spacings between the $l=0$ and $l=2$ modes to be smoother in frequency (this would come at the cost of increasing the frequency uncertainties). We touch briefly on this approach in Sect. 7 below. As far as the (unregularized) fits here are concerned, we feel that it would not be meaningful to quote an average small spacing from the fitted frequencies, given the possibly complicated, and scattered nature of the spacings implied by Fig. 5 .

The large separation derived from the agreed $l=0$ and $l=1$ frequencies is $\Delta v=85.9 \pm 0.15 \mu \mathrm{Hz}$. The new CoRoT estimate differs by more than $6 \sigma$ from the value of $88.7 \mu \mathrm{Hz}$ found by Mosser et al. (2005), from ground-based Doppler velocity observations of HD 49933, but is still in the range of the theoretical large separation given in this latter article. The ground-based spectrum is affected by strong aliases, due to a low duty cycle, and simultaneously a low signal-to-noise ratio, so that the measurement of the large separation may be corrupted by confusion between the ridges of the peaks and those of their aliases.

The other three panels in Fig. 5 show the best-fitting mode linewidths, heights and amplitudes. The mode amplitudes show a peak at $\sim 1760 \mu \mathrm{Hz}$, where the measured rms amplitude for $l=0$ is $3.75 \pm 0.23 \mathrm{ppm}$. The frequency of maximum amplitude for $\mathrm{p}$ modes has been shown to scale with the acoustic cutoff frequency of the star (Bedding \& Kjeldsen 2003). Use of the non-asteroseismic data in Sect. 2 implies an expected frequency of maximum amplitude of $1860 \mu \mathrm{Hz}$, which is to be compared with our measured value of $1760 \mu \mathrm{Hz}$.

We may also make an approximate prediction of the maximum rms amplitude (Kjeldsen \& Bedding 1995) using a $T_{\mathrm{eff}}^{-1 / 2}$ scaling ${ }^{3}$ :

$A_{\max }=(\mathrm{d} L / L)_{\max }=\left(\frac{L / L_{\odot}}{M / M_{\odot}}\right)^{0.7} \sqrt{\frac{5777 \mathrm{~K}}{T_{\text {eff }}}} 2.6 \mathrm{ppm}$.

Here, we have assumed the perturbations due to the modes detected by CoRoT are approximately those in the bolometric luminosity. Furthermore, we make use of the result of Samadi et al. (2007b) who found that the amplitudes in terms of surface velocity scale approximately as $\left[\left(L / L_{\odot}\right) /\left(M / M_{\odot}\right)\right]^{0.7}$. The prediction above is then normalized against the observed maximum amplitude for the Sun, which is about $2.6 \mathrm{ppm}$ rms. The data given in Sect. 2 above imply $\left(L / L_{\odot}\right) /\left(M / M_{\odot}\right) \approx 2.8$ for HD 49933 . Use also of the effective temperature of Bruntt et al. (2008) then

\footnotetext{
3 This scaling based on an adiabatic approximation can also be derived from Berthomieu \& Provost (1990).
} 
gives a predicted maximum rms amplitude of $A_{\max } \approx 4.8 \mathrm{ppm}$. The use of the calibrated scaling of Kjeldsen \& Bedding (1995), which shows an inverse linear dependence on $T_{\text {eff }}$, gives a lower predicted maximum amplitude of $A_{\max } \approx 4.5 \mathrm{ppm}$. In either case, our measured maximum amplitude is significantly lower than the predicted amplitude.

Mosser et al. (2005) have shown that the amplitudes derived from Doppler velocity observations of HD 49933 are also lower than expected. This discrepancy was also found by Mosser et al. (2008) in the case of HD 203608, which is a metal-poor star $([\mathrm{Fe} / \mathrm{H}]=-0.54 \pm 0.03)$. The low metallicity is expected to lower the amplitude of the mode driven by turbulent convection, since it tends to weaken the convective flux in the region where the modes are excited (Samadi et al., 2008; in preparation). Lower mode amplitude could also be the result of the cancellation of the Reynolds stress fluctuations by entropy fluctuations. Indeed, as recently pointed out by Houdek (2006), and as shown by Stein et al. (2004) on the basis of 3D numerical simulations of Sun-like pulsators, entropy fluctuations and Reynolds stress fluctuations can partially interfere. Stein \& Nordlund (2001) showed that the contribution of the entropy fluctuations to the mode driving is rather small in the case of the Sun (of the order of 15\%), a result verified by Samadi et al. (2003). For HD 49933, this contribution is expected to be of the order of $30 \%$, leading to a larger cancellation, and hence lower amplitudes. We should stress that Kjeldsen \& Bedding (1995) already concluded that F-type stars, namely Procyon and several members of M 67, must oscillate with amplitudes less than was generally assumed; their remark was largely based not upon a theoretical work but upon an empirical scaling law that proved to be overestimated.

We may also compare the projected splittings, $v_{\mathrm{s}} \sin i$ (Ballot et al. 2006), given by the asteroseismic and the non-asteroseismic data. The projected splitting for the nonasteroseismic data is $\approx 1.5 \mu \mathrm{Hz}$ for the $3.4 \mu \mathrm{Hz}$ peak. The projected splitting implied by the best-fitting mode splittings and angles of inclination of the seven fitters range from $\approx 2.1 \mu \mathrm{Hz}$ up to $\approx 2.7 \mu \mathrm{Hz}$. The projected splitting given by the Reference fit data is $2.20 \pm 0.02 \mu \mathrm{Hz}$. This projected splitting is also not in agreement with that derived from the non-asteroseimic data. This disagreement is clearly related to the difference between the seismic and non-seismic angles. An underestimation of the $v \sin i$ is rather unlikely as the bisector tends to increase that value. The source of the disagreement may come from an overestimation in the seismic angle due to the closeness of the $l=0$ and $l=2$ modes. This possible bias will be investigated using an approach taking into account the model of the star (Bayesian approach).

\section{Conclusions}

We have analyzed a 60-day lightcurve of high-quality photometric data collected by CoRoT on the F5 V star HD 49933. Despite the obvious presence of a large number of modes in the power spectrum of this lightcurve, the low signal-to-noise ratio leads to some difficulties in the analysis, in particular with regard to the identification of the angular degrees of the modes. We used a likelihood ratio test to discriminate between the possible identifications. The power spectrum of the lightcurve was then fitted to extract estimates of the parameters of the low$l \mathrm{p}$ modes of the star. A global fitting strategy was adopted whereby 15 mode orders spanning almost $1200 \mu \mathrm{Hz}$ of the mode spectrum (45 modes) were fitted simultaneously. Robust results on mode frequencies, linewidths and amplitudes were obtained from a Reference fit for at least 29 modes covering 11 orders.
The most problematic estimate was the mean rotational splitting of the non-radial modes, which appears to be sensitive to the model of the background noise across the range occupied by the modes. The rotational splitting value lies in the range $\approx 2.7 \mu \mathrm{Hz}$ to $\approx 3.4 \mu \mathrm{Hz}$, the lower end indicating a slower than the surface mean rotation, and the higher end indicating a mean rotation of the interior similar to that of the surface. Another difficult estimation is the inclination angle, as determined by fits to the amplitude ratios of the modes, which appears to be in the range $\approx 50$ degrees to $\approx 62$ degrees.

The fittings reported here made no use of a priori constraints from stellar evolution models, or from other prejudice. The analysis performed constituted a classical Maximum Likelihood Estimation (MLE) approach. Given the low heightto-background ratio of the modes, and the large peak linewidths - both of which lead to modest precision in some of the parameters - we feel the next obvious step in the analysis will be to apply techniques that incorporate Bayes theorem, and which therefore use a priori knowledge (e.g., from models, or from other prejudice) to help constrain the best-fitting outputs. For example, fits may be made by applying Maximum A Posteriori (MAP), which is a regularized version of the MLE; or by using MonteCarlo Markov Chains (Appourchaux 2008). We have also been developing these approaches, and the results will be published in future work.

Acknowledgements. IWR and GAV wish to thank the UK Science and Technology Facilities Council for support under grant PP/E001793/1. T.A. thanks the International Space Science Institute (ISSI), where part of this work was done. W.J.C. and Y.E. acknowledge support from the School of Physics and Astronomy at the University of Birmingham. This paper has been approved by the CoRoT Editorial Board.

\section{References}

Alonso, R., Auvergne, M., Baglin, A., et al. 2008, A\&A, 482, L21 Anderson, E. R., Duvall, T. L., J., \& Jefferies, S. M. 1990, ApJ, 364, 699 Appourchaux, T. 2008, Astron. Nachr., 329, 485

Appourchaux, T., Gizon, L., \& Rabello-Soares, M. C. 1998, A\&AS, 132, 107 Appourchaux, T., Berthomieu, G., Michel, E., et al. 2006a, Data analysis tools for the seismology programme The CoRoT Mission, ed. M. Fridlung, A. Baglin, J. Lochard, \& L. Conroy, ESA Publications Division, ESA Spec. Publ., 1306, 377

Appourchaux, T., Berthomieu, G., Michel, E., et al. 2006b, Evaluation of the scientific performances for the seismology programme The CoRoT Mission ed. M. Fridlung, A. Baglin, J. Lochard, \& L. Conroy, ESA Publications Division, ESA Spec. Publ., 1306, 429

Baglin, A. 2006, The CoRoT mission, pre-launch status, stellar seismology and planet finding M. Fridlund, A. Baglin, J. Lochard, \& L.Conroy ed., ESA SP1306 (ESA Publication Division (Noordwijk: The Netherlands)

Ballot, J., García, R. A., \& Lambert, P. 2006, MNRAS, 369, 1281

Ballot, J., Appourchaux, T., Toutain, T., \& Guittet, M., 2008, A\&A, 486, 867

Barge, P., Baglin, A., Auvergne, M., et al. 2008, A\&A, 482, L17

Bedding, T. R., \& Kjeldsen, H. 2003, PASA, 20, 204

Berthomieu, G., \& Provost, J. 1990, A\&A, 227, 563

Bessell, M. S., Castelli, F., \& Plez, B. 1998, A\&A, 333, 231

Bouchy, F., Queloz, D., Deleuil, M., et al. 2008, A\&A, 482, L25

Bruntt, H., De Cat, P., \& Aerts, C. 2008, A\&A, 478, 487

Chaplin, W. J., Appourchaux, T., Baudin, F., et al. 2006, MNRAS, 369, 985

Chaplin, W. J., Houdek, G., Appourchaux, T., et al. 2008, A\&A, 485, 813

Christensen-Dalsgaard, J. 1984, in Space Research in Stellar Activity and Variability, ed. A. Mangeney, \& F. Praderie (Observatoire de Paris), 11 Clarke, D. 2003, A\&A, 407, 1029

De Oliveira Fialho, F., \& Auvergne, M. 2006, The CoRoT Mission ed. M. Fridlung, A. Baglin, J. Lochard, \& L. Conroy, ESA Publications Division, ESA Spec. Publ., 1306, 289

De Oliveira Fialho, F., Lapeyrere, V., Auvergne, M., et al. 2007, PASP, 119, 337 Drummond, R., Vandenbussche, B., Aerts, C., De Oliveira Fialho, F., \& Auvergne, M. 2006, PASP, 118, 874

Duvall, T. L., J., \& Harvey, J. W. 1986, in Seismology of the Sun and the Distant Stars, 105

Gelly, B., Lazrek, M., Grec, G., et al. 2002, A\&A, 394, 285 
Gillon, M., \& Magain, P. 2006, A\&A, 448, 341

Gizon, L., \& Solanki, S. K. 2003, ApJ, 589, 1009

Harvey, J. 1985, in Future missions in solar, heliospheric and space plasma physics, ESA SP-235, ed. E. Rolfe, \& B. Battrick (ESA Publications Division, Noordwijk, The Netherlands), 199

Houdek, G. 2006, [arXiv:astro-ph/0612024]

Kjeldsen, H., \& Bedding, T. R. 1995, A\&A, 293, 87

Libbrecht, K. G. 1992, ApJ, 387, 712

Mangeney, A., Baglin, A., Le Contel, J.-M., et al. 1981, Projet de mission spatiale pour l'étude de la variabilité et de l'activité des étoiles: EVRIS, Etude de la Variabilité, de la Rotation et des Intérieurs Stellaires (Observatoire de Meudon)

Michel, E., Deleuil, M., \& Baglin, A. 2006, The CoRoT Mission ed. M. Fridlung, A. Baglin, J. Lochard, \& L. Conroy, ESA Publications Division, ESA Spec. Publ., 1306, TBD

Mosser, B., Bouchy, F., Catala, C., et al. 2005, A\&A, 431, L13

Mosser, B., Deheuvels, S., Michel, E., et al. 2008, [arXiv:0804. 3119]

Roca Cortés, T., Jiménez, A., Pallé, P. L., GOLF team, \& VIRGO Team. 1999, in Magnetic Fields and Solar Processes, ed. A. Wilson et al., ESA Spec. Publ., 448,135
Roxburgh, I. W. 2006, in ESA Spec. Publ., 1306, 521

Samadi, R., Nordlund, A., Stein, R. F., Goupil, M. J., \& Roxburgh, I. 2003, A\&A, 404, 1129

Samadi, R., Fialho, F., Costa, J. E. S., et al. 2007a, [astro-ph/0703354]

Samadi, R., Georgobiani, D., Trampedach, R., et al. 2007b, A\&A, 463, 297

Schou, J. 1992, Ph.D. thesis, Århus Universitet, Denmark

Solano, E., Catala, C., Garrido, R., et al. 2005, AJ, 129, 547

Stein, R., Georgobiani, D., Trampedach, R., Ludwig, H.-G., \& Nordlund, Å. 2004, Sol. Phys., 220, 229

Stein, R. F., \& Nordlund, Å. 2001, ApJ, 546, 585

Thévenin, F., Bigot, L., Kervella, P., et al. 2006, Mem. Soc. Astron. It., 77, 411

Toutain, T., \& Appourchaux, T. 1994, A\&A, 289, 649

van Leeuwen, F. 2007, Hipparcos, the New Reduction of the Raw Data (Hipparcos, the New Reduction of the Raw Data. By Floor van Leeuwen, Institute of Astronomy, Cambridge University, Cambridge, UK Series: Astrophysics and Space Science Library, 350, 20 (Dordrecht: Springer) 